\title{
Restoring particle phenomenology
}

\author{
Giovanni Valente \\ Cathedral of Learning, 1001 CL, Pittsburgh, PA 15260, United States
}

\section{ABSTRACT}

No-go theorems are known in the literature to the effect that, in relativistic quantum field theory, particle localizability in the strict sense violates relativistic causality. In order to account for particle phenomenology without particle ontology, Halvorson and Clifton (2002) proposed an approximate localization scheme. In a recent paper, Arageorgis and Stergiou (2013) proved a no-go result that suggests that, even within such a scheme, there would arise act-outcome correlations over the entire spacetime, thereby violating relativistic causality. Here, we show that this conclusion is untenable. In particular, we argue that one can recover particle phenomenology without having to give up relativistic causality.

\section{Introduction}

Quantum field theory is our best candidate for a relativistic version of quantum mechanics. Although it has been confirmed experimentally, the jury is still out as to what the fundamental constituents of matter it purports to describe really are. In principle, its ontology would lend itself to either a particle interpretation or a field interpretation. Yet, underlying much of contemporary experimental physics aiming at testing the theory there seems to be a particle ontology, in that one employs procedures designed for the detection of particles: for instance, scattering experiments presuppose the existence of particle trajectories. In fact, the phenomenology of quantum field theory appears as a particle phenomenology. A necessary condition for particle ontology is that particles possess a position, so that they can actually be localized within some spacetime region where a detector is set up. Nevertheless, various no-go theorems against particle localization in relativistic quantum theory have been proven (Hegerfeldt, 1998; Malament, 1996; Redhead, 1995; Reeh \& Schlieder, 1961). Accordingly, a particle ontology would become untenable. If so, though, one still ought to account for the observed experimental phenomena, which appear as particle detections. That is, how can one sustain a particle phenomenology without particle ontology?

E-mail address: valente@pitt.edu
Halvorson and Clifton (2002) proved a series of no-go results generalizing the previous theorems against particle localization: under mild relativistic constraints, the concept of localizability can be shown to be in conflict with the requirement of relativistic causality, taken more precisely as the condition of microcausality (also referred to as Einstein's principle of causality). They take this as ruling out a particle interpretation of quantum field theory. Then, in the attempt to "salvage the appearances" observed in the laboratory, they developed a procedure to account for particle phenomenology in Algebraic Quantum Field Theory based on an approximate localization scheme resorting to the notion of almost local observables, which, they say, one can adopt for all practical purposes. In an interesting recent paper, Arageorgis and Stergiou (2013) cast Halvorson and Clifton's scheme within the framework of "minimally statistically faithful particle detection experiments", and they proved a no-go theorem that suggests that appealing to almost local observables is still at variance with relativistic causality, in that measurements of such observables would entail act-outcome correlations over the entire spacetime. Here, we show that this conclusion is ungrounded. In fact, we claim that, although Arageorgis and Stergiou's result is certainly correct and deserves close attention, it does not raise any conflict with relativistic causality, nor any threat of act-outcome correlations. In particular, we wish to argue that one can still recover particle phenomenology without having to give up relativistic causality.

We first recall the notion of microcausality and its connection with the absence of act-outcome correlations in Algebraic 
Quantum Field Theory, and we spell out the consequences of the Reeh-Schlieder theorem for the problem of particle localizability (Section 2.1). Then, we review Halvorson and Clifton's attempt to salvage particle phenomenology without particle ontology (Section 2.2). In the following section, we present Arageorgis and Stergiou's no-go result together with the interpretation proposed by the authors. In Section 4 we show that their theorem is not in contradiction with relativistic causality taken as microcausality; whereas in Section 5 we explain in what sense it does not entail act-outcome correlations extending over the entire spacetime. Finally, we conclude by using our analysis so as to answer the question Arageorgis and Stergiou raised in the title of their paper, that is "How Much Local is Almost Local?".

\section{Particle phenomenology without particle ontology}

\subsection{Localizability and relativistic causality}

Halvorson and Clifton (2002) no-go theorems support arguments against the possibility of localizing a single particle, where localizability is formalized in terms of a position operator associated with some bounded region of space, no matter how large. Algebraic Quantum Field Theory offers the prospect to capture spacetime localizability in a different way. In fact, in this framework relativistic quantum field systems are described by mapping $\mathcal{O} \longmapsto \mathcal{R}(\mathcal{O})$ sending any bounded region $\mathcal{O}$ of Minkowski spacetime $M$ to the local algebra $\mathcal{R}(\mathcal{O})$ defined on the underlying Hilbert space $\mathcal{H}$ containing all the observables one can measure within the corresponding region. Any observable $A \in \mathcal{R}(\mathcal{O})$ is said to be a local observable localized in $\mathcal{O}$. This grounds the possibility to introduce an alternative localization scheme: accordingly, a "click" in a particle detector within an arbitrarily small spacetime region is modeled by some local observable belonging to the corresponding local algebra. The standard axioms of Algebraic Quantum Field Theory are presented in Haag (1992). Here, we just note that they guarantee that the local algebras $\mathcal{R}(\mathcal{O})$ are von Neumann algebras and that the inductive limit of the net of all local algebras is given by the quasi-local $C^{*}$-algebra $\mathcal{R}=\overline{\{\mathcal{R}(\mathcal{O}) \mid \mathcal{O} \subset M\}}$. In particular, the vacuum state is represented by the normalized vector $\Omega$, which is the unique Poincare invariant vector-state.

The condition of relativistic causality assumed in the no-go theorems against particle localizability is captured by the axiom of microcausality. For simplicity, let us refer to diamond regions: specifically, a double cone $\mathcal{O}$ in $M$ satisfies the diamond axiom just in case the local algebra associated with its causal complement $\mathcal{O}^{\prime}$ is equal to the commutant of its local algebra, i.e. $\mathcal{R}\left(\mathcal{O}^{\prime}\right)=\mathcal{R}(\mathcal{O})^{\prime}$. Microcausality requires that any local observable in $\mathcal{R}(\mathcal{O})$ commutes with all the local observables in $\mathcal{R}\left(\mathcal{O}^{\prime}\right)$. The physical idea here is that it assures that measurements of observables localized in region $\mathcal{O}$ do not disturb measurements of observables localized in its causal complement $\mathcal{O}^{\prime}$. The justification for regarding microcausality as an expression of relativistic causality is given in terms of the constraint of no superluminal signalling. Since $\mathcal{O}$ and $\mathcal{O}^{\prime}$ are spacelike separated, a measurement performed in the former region ought not to cause an instantaneous change of the expectation value of any observable localized in the latter region, regardless of what the state of the system is. This idea can be made precise by means of the Lüders rule describing quantummechanical measurements. A generalized Lüders rule for effects has been formulated by Busch and Singh (1998) and Busch (1999), where an effect is any positive observable $C$ whose expectation value $\langle\psi \mid C \psi\rangle$ lies in $[0,1]$ for every unit vector $\psi$, so that $\|C\| \leq 1$ (see Kraus, 1983 for a review). On contrary to projections, effects allow one to account even for measurements with unsharp outcomes. In its simplest form the measurement of an effect $C$ can be represented by the operation

$T_{C}(\cdot)=C^{1 / 2}(\cdot) C^{1 / 2}+(I-C)^{1 / 2}(\cdot)(I-C)^{1 / 2}$

It can be shown that, just in case microcausality holds, if $C$ belongs to $\mathcal{R}(\mathcal{O})$ the thus-defined operation $T_{C}$ acts as the identity in $\mathcal{R}\left(\mathcal{O}^{\prime}\right)$, thereby leaving invariant all observables localized in the causal complement of $\mathcal{O}$. As a consequence, $T_{C}$ does not change the expectation value of any observable $A$ in $\mathcal{R}\left(\mathcal{O}^{\prime}\right)$, that is $\langle\psi \mid A \psi\rangle=\left\langle\psi \mid T_{C}(A) \psi\right\rangle$ for every vector-state $\psi \in \mathcal{H}$. Accordingly, a measurement of $C$ does not have non-local effects in $\mathcal{O}^{\prime}$. That yields a version of the no-superluminal-signalling theorem in Algebraic Quantum Field Theory, whereby a signal is enacted by the Lüders rule (1). Failure to comply with such a requirement would thus result in a violation of no faster-than-light signaling. Let us emphasize that this would also entail the presence of actoutcome correlations across the field systems within the region $\mathcal{O}$ and its causal complement $\mathcal{O}^{\prime}$. The notion of act-outcome correlations has been widely discussed in the philosophical literature on quantum non-locality. Indeed, Shimony (1986) associated it with a violation of the condition of parameter independence in the decomposition of Bell-type locality, thereby leading to a conflict with the constraint of relativistic causality.

Although the concept of localization of observables in spacetime regions is compatible with microcausality, from the axioms of Algebraic Quantum Field Theory one can derive a theorem that poses a threat for particle localizability. That is the Reeh-Schlieder theorem (1961): accordingly, for all regions $\mathcal{O}$ in Minkowski spacetime $M$, any vector-state of bounded energy, such as the vacuum $\Omega$, is cyclic for the local algebras $\mathcal{R}(\mathcal{O})$. The vacuum being cyclic means that, for every vector $\psi \in \mathcal{H}$, there exists a sequence of observables $\left\{F_{n}\right\}_{n \in N}$ in $\mathcal{R}(\mathcal{O})$ such that

$\left\|\psi-F_{n} \Omega\right\| \longrightarrow 0$

when $n$ goes to infinity. In other words, one is able to approximate in norm any vector-state by acting on the vacuum with observables localized in the bounded region $\mathcal{O}$. In particular, one could generate a state which looks within the causal complement $\mathcal{O}^{\prime}$ very different from $\Omega$. Whether it entails a violation of relativistic causality is an outstanding issue in philosophy of physics (see Valente, 2014 for a recent discussion). However, as Fleming and Butterfield (1999) observed, "that is certainly hard to square with naive, or even educated, intuitions about localization!" (p. 159). In fact, Redhead (1995) showed that, owing to the Reeh-Schlieder theorem, one can never determine whether the system is in an $\mathrm{N}$-particle state since the corresponding projection operator cannot belong to any local algebra. ${ }^{1}$

There is also another crucial reason why the Reeh-Schlieder theorem would undermine the intended localization scheme in Algebraic Quantum Field Theory. A necessary condition for a particle ontology is that one cannot detect any particle in the vacuum. Thus, the expectation value of a putative observable $C$ designed to model a particle detection must be zero in the vacuum. Let us refer to it as the following:

$$
\text { Condition (I): }\langle\Omega \mid C \Omega\rangle=0
$$

Yet, in conjunction with microcausality, the Reeh-Schlieder theorem entails the corollary that the vacuum is a separating vector for any local algebra associated with a region with nonempty causal complement. It means that, if $\mathcal{O}^{\prime} \neq \varnothing$, then for all $C \in \mathcal{R}(\mathcal{O})$ one has $C \Omega=0$ just in case $C=0$. It follows that the expectation value of any non-trivial observable $C$ localized in

\footnotetext{
${ }^{1}$ Actually, the proof of Redhead's result appeals also to the fact that all projections in the local algebras, being type III factors von Neumann algebras, are infinite. Since it is not relevant to our discussion, due to length constraints, we do not introduce this notion here.
} 
region $\mathcal{O}$ is always non-zero in the vacuum vector-state, i.e. $\langle\Omega|$ $C \Omega\rangle \neq 0$. Indeed, no local observable can annihilate the vacuum. Therefore, Condition (I) fails to hold. It is the violation of this requirement that motivates one to resort to approximate localization schemes, such as the one developed by Halvorson and Clifton (2002).

Before turning to this proposal, though, we wish to discuss another condition for particle localization. That is that a particle can actually be detected in some state different from the vacuum. Accordingly, for some state other than $\Omega$, the observable $C$ has a non-zero expectation value. Let us formalize such a requirement as follows:

Condition (II): There exists some vector-state $\psi \neq \Omega$ such that $\langle\psi \mid C \psi\rangle \neq 0$

This actually seems a rather reasonable desideratum. The more so because, if it fails, the observable $C$ has zero expectation value for all states $\psi \neq \Omega$ : therefore, unless its expectation value in the vacuum $\Omega$ is non-zero, it would follow that $C=0$. Nevertheless, it proves at variance with Condition (I) above, due to the Reeh-Schlieder theorem. In fact, if a local observable $C$ annihilates the vacuum, then it annihilates any other state, thereby violating Condition (II). For, suppose $C$ is localized in the bounded region $\mathcal{O}$ : if one considers Eq. (2)'s sequence of observables $\left\{F_{n}\right\}_{n \in N}$ belonging to the local algebra $\mathcal{R}\left(\mathcal{O}^{\prime}\right)$, then

$$
\begin{aligned}
& \left\|\psi-F_{n} \Omega\right\| \geq\|C\| \cdot\left\|\psi-F_{n} \Omega\right\| \\
& \left\|\psi-F_{n} \Omega\right\| \geq\left\|C\left(\psi-F_{n} \Omega\right)\right\| \\
& \left\|\psi-F_{n} \Omega\right\|=\left\|C \psi-C F_{n} \Omega\right\| \\
& \left\|\psi-F_{n} \Omega\right\| \stackrel{M C}{=}\left\|C \psi-F_{n} C \Omega\right\| \\
& \left\|\psi-F_{n} \Omega\right\| \stackrel{\text { Cond(I) }}{=}\|C \psi\|
\end{aligned}
$$

The first inequality is due to $\|C\| \leq 1$, whereas the second is a consequence of the Cauchy-Schwarz inequality. In step (5) one employs microcausality, which guarantees that $C \in \mathcal{R}(\mathcal{O})$ commutes with all the $F_{n}$ 's. The last equation follows from Condition (I), in that it implies $C \Omega=0$. Finally, by the cyclicity of the vacuum for $\mathcal{R}\left(\mathcal{O}^{\prime}\right)$ assured by the Reeh-Schlieder theorem and Eq. (2), $\| \psi$ $-F_{n} \Omega \| \longrightarrow 0$ as $n$ tends to infinity, and hence also $\|C \psi\|$ has to vanish. One thus concludes that $\langle\psi, C \psi\rangle=0$, in flat contradiction with Condition (II). We shall come back to the interplay between these two conditions for particle localizability when discussing the Arageorgis-Stergiou theorem.

\section{2. "Salvaging the appearances": almost local observables}

In order to cope with the problem that no non-zero local observable can annihilate the vacuum, Halvorson and Clifton developed a procedure resorting to the notion of almost local observables. Their proposal is not novel in that it draws from previous authors, most notably Haag (1992) and Buchholz (2000), who provided a more elaborated characterization of particle detectors. Yet, Halvorson and Clifton offered it as a way to maintain a particle phenomenology without particle ontology, thereby "salvaging the appearances" observed in the experimental context of particle detectors. It is based on an approximate localization scheme which, they say, one can adopt "for all practical purposes".

The starting point of the purported account of particle phenomenology is that, for any observable $C$, one can find a class of observables $C^{\prime}$ that approximate $C$ in norm, in the sense that

$\left\|C^{\prime}-C\right\|<\delta$ for any arbitrarily small positive real number $\delta$. By the definition of the operator norm as the supremum of $\left\|\left(C^{\prime}-C\right) \psi\right\|$ for all unit vectors $\psi$, this means that $C^{\prime}$ and $C$ dictate close expectation values in any vector-state: in fact, the CauchySchwarz inequality implies that $\left\|\left(C^{\prime}-C\right) \psi\right\|=\|\psi\| \cdot\left\|\left(C^{\prime}-C\right) \psi\right\|$ is greater or equal than $\left|\left\langle\psi \mid\left(C^{\prime}-C\right) \psi\right\rangle\right|=\left|\left\langle\psi \mid C^{\prime} \psi\right\rangle-\langle\psi \mid C \psi\rangle\right|$, and therefore the latter must be strictly smaller than $\delta$ as well. Accordingly, measurements of $C$ and $C^{\prime}$ are practically indistinguishable in any vector-state $\psi$. This view is further enforced by the idea that measurements always involve errors and environmental noise, so that one cannot expect to determine precisely what observable in the relevant equivalence class determined by the norm distance is effectively measured. To put it formally, for a fixed arbitrarily small $\delta$, let the set of operators

$\mathcal{R}_{\delta}(\mathcal{O})=\left\{C^{\prime} \mid \exists C \in \mathcal{R}(\mathcal{O})\right.$ such that $\left.\left\|C^{\prime}-C\right\|<\delta\right\}$

denote the class of observables $C^{\prime}$ being "almost localized" in $\mathcal{O}$, in that each of them approximates in norm some observable $C$ localized in $\mathcal{O}$. Then, as Halvorson and Clifton explained,

"FAPP" (i.e., "for all practical purposes") we can locally measure any observable from $\mathcal{R}_{\delta}(\mathcal{O})$. That is, measurement of an element from $\mathcal{R}_{\delta}(\mathcal{O})$ can be simulated to a high degree of accuracy by local measurement of an element from $\mathcal{R}(\mathcal{O})$. [p. 22]

The next step to complete the suggested approximate localization scheme is to identify an operator $\bar{C}$ contained in the set $\mathcal{R}_{\delta}(\mathcal{O})$ that annihilates the vacuum. That is defined as an almost local observable. The existence of the sought-after operator was shown by Buchholz (2000). We defer its explicit construction to Section 5 . Here, we just point out that it is taken to be an element of the quasi-local algebra $\mathcal{R}$. The upshot is that, among the observables $C^{\prime}$ contained in $\mathcal{R}_{\delta}(\mathcal{O})$ that approximate in norm a local observable $C \in \mathcal{R}(\mathcal{O})$, there is an almost local observable $\bar{C}$ such that $\langle\Omega, \bar{C} \Omega\rangle=0$. In light of this, Halvorson and Clifton could conclude that

particle detections can always be simulated by purely local measurements; and we can explain the appearance of macroscopically localized objects without assuming that there are localizable particles in the strict sense. [p. 22]

By adopting such an approximate localization scheme holding for all practical purposes, one is thus in a position to recover particle phenomenology without particle ontology. However, Arageorgis and Stergiou (2013) argued that, even within such a scheme, there would arise a conflict with relativistic causality, in that almost local observables would entail act-out correlations, thereby falling back into the problems besetting the standard schemes for particle localizability in the strict sense.

\section{Arageorgis and Stergiou's argument}

Arageorgis and Stergiou discussed Halvorson and Clifton's proposal within the framework of effects. Accordingly, the almost local observable $\bar{C}$ is assumed to be an effect, corresponding to a positive operator in the quasi-local algebra $\mathcal{R}$ such that $\|\bar{C}\| \leq 1$. The purported approximate localization scheme is then cast in terms of "minimally statistically faithful particle detection experiments":

the notion of minimally statistically faithful particle detection experiment involves a positive observable whose measurement gives no response in the vacuum state but a positive response in at least one other state... [O]f course, the said observable must be somehow "macroscopically well-enough-localized". [p. 972] 
The provision in the first sentence requires that the almost local observable $\bar{C}$ satisfies not only Condition (I), but also Condition (II) stated in Section 2.1. In other words, while annihilating the vacuum, $\bar{C}$ ought to have a non-zero expectation value in some vector-state different from $\Omega$. Notice that the argument we previously formulated to the effect that Condition (I) and Condition (II) are incompatible does not apply here: in fact, there is no appeal to microcausality at the present stage, and hence the inference to Eq. (5) is not valid. So, in principle, almost local observables may annihilate the vacuum without necessarily annihilating all other states. It should be stressed, though, that the last clause in the above quotation, expressing the desideratum that the putative observable $\bar{C}$ ought to be "macroscopically well-enoughlocalized", is somewhat ambiguous. As we argue in Section 5, this actually undermines Arageorgis and Stergiou's analysis. However that may be, the authors proceeded to prove the following result.

Arageorgis-Stergiou Theorem: There is no positive observable $\bar{C} \in \mathcal{R}$ such that $\|\bar{C}\| \leq 1$, which satisfies the following conditions:

- Condition (I): $\langle\Omega \mid \bar{C} \Omega\rangle=0$ for the vacuum vector $\Omega$;

- Condition (II): there is at least one vector-state $\psi \neq \Omega$ such that $\langle\psi \mid \bar{C} \psi\rangle \neq 0$

- Condition (III): there exists at least one region $\mathcal{O}$ of Minkowski spacetime $M$ such that, given any vector-state $\psi$ and for all $A \in \mathcal{R}(\mathcal{O}),\langle\psi \mid A \psi\rangle=\left\langle\psi \mid T_{\bar{C}}(A) \psi\right\rangle$, where the operation $T_{\bar{C}}$ is defined by (1)

Allegedly, owing to this theorem, the notion of minimally statistically faithful particle detection experiment would entail act-outcome correlations over the entire spacetime.

The rationale for such a claim rests on Arageorgis and Stergiou's interpretation of the third condition they invoked in their theorem. Condition (III) demands that the operation $T_{\bar{C}}$ representing a measurement of the effect $\bar{C}$ via the generalized Lüders rule (1) for effects does not change the expectation value of any observable localized in some region $\mathcal{O}$ of Minkowski spacetime, regardless of the state the system is in. A straightforward calculation shows that it implies that $T_{\bar{C}}$ acts as the identity on the local algebra associated with the given region $\mathcal{O}$, that is $T_{\bar{C}}(A)=A$ for any local observable $A \in \mathcal{R}(\mathcal{O})$. Agreed, such a constraint seems to require very little: in order for it to be fulfilled, it is sufficient that there is even just one region of spacetime over which $T_{\bar{C}}$ behaves in the desired way. At first sight, that may indeed look like a minimal requirement of causality. So, its failure would have radical consequences, namely that, for all regions $\mathcal{O}$ of Minkowski spacetime, there is always some local observable in each local algebra $\mathcal{R}(\mathcal{O})$ whose expectation value computed in some state is changed when the operation $T_{\bar{C}}$ is performed. Accordingly, if one interprets the fact that a measurement of $\bar{C}$ does not leave invariant the expectation values of all observables localized in $\mathcal{O}$ as an instance of act-outcome correlations, a violation of Condition (III) would entail a-causal holistic effects, in that it would establish actoutcome correlations over the entire spacetime.

In fact, on the basis of the present interpretation, Arageorgis and Stergiou concluded that

the feigned particle detectors, even if acceptable "for all practical purposes" as to the desideratum of localizability, would still have "bad" behavior as to the desideratum of relativistic causality. For they would probe radically holistic features of the quantum field by establishing act-outcome correlations over the entire spacetime. [pp. 975-976]
It would thus seem that a conflict with relativistic causality, which underlies the no-go theorems against particle localizability in the strict sense, presents itself again when resorting to almost local observables to represent particle detectors. Such a claim lends itself to two possible readings. For one, Arageorgis and Stergiou's result suggests yet another argument against a particle ontology in relativistic quantum field theory: if the physical possibility of minimally statistically faithful particle detections is a necessary condition for a particle ontology, then the latter is admissible only at the cost of allowing for act-outcome correlations over the entirety of spacetime. That is the position the authors endorse explicitly towards the end of the paper. One the other hand, and perhaps more dramatically, their theorem can be even understood as undercutting the attempt to account for particle phenomenology outlined in Section 2.2. In fact, it is fully formulated in the context of Halvorson and Clifton's approximate localization scheme. Accordingly, one may hope to salvage the appearances observed in the laboratory just if particle detections are described in terms of observables with causally badly-behaving properties. But is there really a violation of relativistic causality, or any sort of act-outcome correlations over the entire spacetime, as Arageorgis and Stergiou maintain?

We wish to argue that such a claim is ungrounded. Our analysis proceeds as follows. First of all, we show that Arageorgis and Stergiou's theorem does not involve microcausality. As such, it cannot raise a conflict with the condition of relativistic causality. We take this up in the next section. Then, in Section 5, we observe that their intended notion of act-outcome correlations is not welldefined, in that almost local observables lack a suitable spacetime localization. Moreover, we stress that, in the present context, such observables ought to be regarded as mere fictions, and hence, even if they could entail some kind of act-outcome correlations, that would not have any real physical significance.

\section{Is there really a conflict with relativistic causality?}

Recall that in the no-go results against particle localizability in the strict sense microcausality is a crucial assumption. In fact, it is this axiom that is accorded the status of relativistic causality, in that it entails a no-superluminal-signalling theorem: if a quantum measurement is represented by the Lüders rule (1), then no measurement of any observable performed in a region $\mathcal{O}$ can have non-local effects within its causal complement, in the sense that it leaves invariant the expectation values of all observables localized in $\mathcal{O}^{\prime}$, no matter what the initial state of the system is. Arguably, the attempt to localize a particle leads to a violation of relativistic causality. However, we now show explicitly that Arageorgis and Stergiou's no-go result does not rest on microcausality at all.

To see this point, let us look in detail at the proof of their theorem. Specifically, it appeals to the following purely algebraic Lemma, which applies to operations of the form $T_{C}$.

Lemma: If $C$ is an effect on a Hilbert space $\mathcal{H}$ such that $T_{C}(A)=A$ for every self-adjoint operator $A$ in a concrete $C^{*}$-algebra $\mathcal{A}$ on $\mathcal{H}$, then $C^{1 / 2}$ commutes with every element of $A$, i.e. $\left[C^{1 / 2}, F\right]=0$ for all $F \in \mathcal{A}$.

Such a Lemma guarantees that, whenever $T_{C}$ acts as the identity in the algebra $\mathcal{A}$, the operator $C^{1 / 2}$ in the decomposition of the said operation given by the Lüders rule (1) commutes with any operator in the algebra. Now, assuming that the almost local observable $\bar{C}$ obeys Condition (III), the operation $T_{\bar{C}}$ acts as the identity on the local algebra $\mathcal{R}(\mathcal{O})$ associated with a given bounded region $\mathcal{O}$. Hence, by the Lemma, the operator $\bar{C}^{1 / 2}$ in the decomposition of $T_{\bar{C}}$ commutes with all observables localized in $\mathcal{O}$. 
The Arageorgis-Stergiou theorem exploits this fact to show that, if Condition (III) holds, one derives a contradiction between Condition (I) and Condition (II) for the almost local observable $\bar{C}$. The proof closely resembles that given in Section 2.1 to demonstrate that Condition (I) implies a violation of Condition (II) for any local observable $C$. Let us suppose that $\bar{C}$ satisfies Condition (III): then, if one considers the sequence of observables $\left\{F_{n}\right\}_{n \in N}$ belonging to the local algebra $\mathcal{R}(\mathcal{O})$ as in Eq. (2), one obtains

$$
\begin{aligned}
& \left\|\psi-F_{n} \Omega\right\| \geq\left\|\bar{C}^{1 / 2}\right\| \cdot\left\|\psi-F_{n} \Omega\right\| \\
& \left\|\psi-F_{n} \Omega\right\| \geq\left\|\bar{C}^{1 / 2}\left(\psi-F_{n} \Omega\right)\right\| \\
& \left\|\psi-F_{n} \Omega\right\|=\left\|\bar{C}^{1 / 2} \psi-\bar{C}^{1 / 2} F_{n} \Omega\right\| \\
& \left\|\psi-F_{n} \Omega\right\| \stackrel{\text { Lemma }}{=}\left\|\bar{C}^{1 / 2} \psi-F_{n} \bar{C}^{1 / 2} \Omega\right\| \\
& \left\|\psi-F_{n} \Omega\right\| \stackrel{\operatorname{Cond}^{\prime}(I)}{=}\left\|\bar{C}^{1 / 2} \psi\right\|
\end{aligned}
$$

Notice that, for any vector-state $\psi$, it follows that $\bar{C}^{1 / 2} \psi=0$ just in case $\bar{C} \psi=0$. So, Condition (I) implies $\bar{C}^{1 / 2} \Omega=0$, thereby justifying the last inference to (12). But, by the Reeh-Schlieder theorem, the vacuum vector is cyclic for $\mathcal{R}(\mathcal{O})$, and hence $\left\|\psi-F_{n} \Omega\right\| \longrightarrow 0$ when $n$ goes to infinity. As a consequence, $\left\|\bar{C}^{1 / 2} \psi\right\|$ has to vanish as well. This argument can be repeated for any vector-state $\psi$ (with concordant change in the choice of the sequence $\left(\left\{F_{n}\right\}_{n \in N}\right)$, so that we get to a contradiction with Condition (II) for the almost local observable $\bar{C}$ ).

Modulo the use of $\bar{C}^{1 / 2}$ rather than $C$, the only difference from the derivation in Section 2.1 is that one uses the Lemma instead of microcausality in order to justify the inference to Eq. (11). Here, one does not need microcausality to assure that $\bar{C}$ commutes with any operator $F_{n}$ in $\mathcal{R}(\mathcal{O})$. This axiom is not invoked at all, and indeed it plays no role. Furthermore, it is not implied by Condition (III) and the Lemma. Nor does it follow from any other assumption in Arageorgis and Stergiou's theorem. Yet, on the basis of the alleged conflict with relativistic causality, they offered their result as "an independent shortcut to an argument" against a particle ontology in Algebraic Quantum Field Theory. As they put it:

We say "shortcut to an argument" because the crux of the issue -namely, the tension between localizability and relativistic causality-is inherent already in the celebrated Reeh-Schlieder theorem that underlies our proof. (p. 974)

Their proof does indeed rely on the Reeh-Schlieder theorem. Nevertheless, such a theorem is provably not a consequence of microcausality: in fact, in order to prove it one needs only isotony, relativistic covariance and weak-additivity (see Halvorson, 2001; Valente, 2014). To be sure, the standard no-go results against particle localizability based on the Reeh-Schlieder theorem assume the requirement that the local algebra $\mathcal{R}(\mathcal{O})$ commutes with $\mathcal{R}\left(\mathcal{O}^{\prime}\right)$, that is needed to demonstrate that the vacuum is a separating vector for $\mathcal{R}(\mathcal{O})$ when the causal complement $\mathcal{O}^{\prime}$ of $\mathcal{O}$ is non-empty. However, this corollary of the Reeh-Schlieder theorem is not utilized in the above derivation. Instead, the latter appeals just to the property that the vacuum vector $\Omega$ is cyclic for $\mathcal{R}(\mathcal{O})$, which is fully independent from microcausality. So, in the last analysis, one cannot infer from Arageorgis and Stergiou's theorem that the attempt to localize a particle, even in the non-strict sense captured by Halvorson and Clifton's approximate localization scheme, violates microcausality. The claim that putative particle detectors described by almost local observables such as $\bar{C}$ would have bad behavior as regards relativistic causality is therefore ungrounded, inasmuch as the axiom of microcausality is interpreted as expressing the condition of relativistic causality.
One should also stress that Arageorgis and Stergiou's result rests on the choice of the Lüders rule for effects as the appropriate representation of a quantum measurement. Supposedly, the operation $T_{\bar{C}}$ maps the quasi-local-algebra $\mathcal{R}$ onto itself. However, there exist plenty of other operations of a different form than $T_{\bar{C}}$, depending on their domain of definition. For such maps the Lemma does not hold since they do not admit the decomposition (1), and hence the proof of the theorem would not go through as it stands. This clearly restricts the scope of Arageorgis and Stergiou's argument.

On the other hand, the authors pointed out that general operations constructed by Rédei and Valente (2010) in Algebraic Quantum Field Theory may also violate the constraint of no superluminal signalling. Yet, Rédei and Valente also showed that one can always explain away the ensuing non-local effects by invoking a strengthening of microcausality, that is the split property. Moreover, contrary to $T_{\bar{C}}$, these operations are defined on the joint algebra generated by the local algebras associated with a pair of spacelike separated regions, rather than on the quasi-local algebra $\mathcal{R}$, and therefore they can be ascribed a welldefined localization in spacetime. In our view, the lack of spacetime localization of almost local observables such as $\bar{C}$ yields cogent reasons to reject Arageorgis and Stergiou's claim that the putative particle detectors in Halvorson and Clifton's approximate localization scheme entail act-outcome correlations. Let us turn to this issue in the following section.

\section{Almost local observables are not localized}

The fact that Arageorgis and Stergiou's theorem does not imply that a conflict with microcausality would arise when one attempts to localize a particle has direct consequences for the claim that there are act-outcome correlations over the entire spacetime. As we previously explained, the absence of act-outcome correlations at spacelike distance is connected to microcausality via a nosuperluminal-signalling theorem using the Lüders rule (1). Inasmuch as the axiom of microcausality is compatible with all the conditions employed in Arageorgis and Stergiou's result, there can be no act-outcome correlations arising from the latter either. Yet, one may claim that the alleged violation of relativistic causality stems from the failure of Condition (III) in the theorem, rather than from a conflict with microcausality. In this vein, the Arageorgis-Stergiou theorem would suggest a "shortcut" to an alternative argument against particle ontology than the standard no-go theorems.

In order to assess this claim, let us reformulate the content of their theorem as a conditional statement: for any positive observable $\bar{C} \in \mathcal{R}$ such that $\|\bar{C}\| \leq 1$, if it satisfies Condition (I) and Condition (II), then it violates Condition (III). In other words, if the putative almost local observable $\bar{C}$ annihilates the vacuum but has a non-zero expectation value in some other state, there is no region $\mathcal{O}$ of Minkowski spacetime for which a measurement of $\bar{C}$ leaves invariant the expectation values of all local observables in $\mathcal{R}(\mathcal{O})$ as computed in any vector-state. Arguably, Arageorgis and Stergiou's claim that the failure of Condition (III) would entail acausal holistic effects depends on the interpretation of the change of expectation values of some observables, at least one for each bounded spacetime region, by means of the operation $T_{\bar{C}}$ as an instance of act-outcome correlations. However, such an interpretation is unwarranted, for the almost local observable $\bar{C}$ does not have any spacetime localization. Indeed, owing to the ReehSchlieder theorem, such an almost local observable cannot be an observable localized in any spacetime region, else one would violate Condition (I). Instead, $\bar{C}$ must lie in the subset $\mathcal{R} / \bigcup_{\mathcal{O} \subset M} \mathcal{R}(\mathcal{O})$ of operators in $\mathcal{R}$ that do not belong to any local 
algebra $\mathcal{R}(\mathcal{O})$. Thus, it is not even clear where the measurement of $\bar{C}$ represented by the operation $T_{\bar{C}}$ would take place.

The notion of act-outcome correlations is usually understood as a relation between quantum systems within spacelike separated regions. Yet, since almost local observables are not localized, the supposed "act" associated with the measurement of an almost local observable is not taking place anywhere, or at least not in any specific region in Minkowski spacetime. As a consequence, one of the relata remains un-instantiated, even if the "act" would seem to have effects in all bounded spacetime regions due to the violation of Condition (III) in Arageorgis and Stergiou's theorem. In light of this, the assertion that there are act-outcome correlations over the entire spacetime appears to be meaningless in the context of almost local observables. Therefore, the alternative argument against a particle ontology in relativistic quantum field theory suggested by Arageorgis and Stergiou loses its force.

One could perhaps hope to mitigate such an objection by holding the view that, although the almost local observable $\bar{C}$ is not, strictly speaking, localized in spacetime, it is still somehow "macroscopically well-enough-localized", as the last clause in the quotation from Arageorgis and Stergiou at the beginning of Section 3 intimates. Indeed, since $\bar{C}$ is taken from the set $\mathcal{R}_{\delta}(\mathcal{O})$ of operators that approximate in norm some observable in $\mathcal{R}(\mathcal{O})$, there would seem to be a sense in which the putative spacetime localization of $\bar{C}$ is within the region $\mathcal{O}$. Nevertheless, the proof of the existence of the sought-after almost local observables by Buchholz (2000, pp. 7-8) indicates that even such a more relaxed notion of "macroscopically well-enough-localized" observables is hard to sustain. Buchholz's construction is as follows. Given any local observable $C$ in $\mathcal{R}(\mathcal{O})$, one can define the operator $C(x)=U(x) C U(x)^{*}$, where $U(x)$ denote the unitaries representing the spacetime translations by $x \in R^{4}$ in Minkowski spacetime. Then, one can construct an almost local observable by smearing the spacetime translates of $C$ with an appropriate test function ${ }^{2} f$ defined on $M$ : for, Buchholz showed that, if the Fourier transform of $f$ has a compact support outside the forward light cone, the following operator:

$L \stackrel{\text { def }}{=} C(f) \stackrel{\text { def }}{=} \int f(x) C(x) d x$

annihilates the vacuum. In order to obtain a positive almost local operator, one can further stipulate $\bar{C}=L^{*} L$. The point we wish to make is that, even though the observable $\bar{C}$ in $\mathcal{R}_{\delta}(\mathcal{O})$ is indeed constructed out of an observable $C$ in $\mathcal{R}(\mathcal{O})$, it is obtained by translating the latter outside the original region $\mathcal{O}$ over the rest of Minkowski spacetime. Therefore, the operator $\bar{C}$ arises, so to speak, as a "global" observable. As such, it would not quite lend itself to be conceived as being well-enough-localized within some bounded spacetime region.

Be that as it may, there is a further reason why one can deny that almost local observables entail act-outcome correlations, which depends on the assumption that the real observables for quantum fields ought to have a well-defined spacetime localization. As Arageorgis and Stergiou themselves conceded,

one may plausibly claim that to act on almost local observables is just another fiction: every physically realistic operation takes place in a bounded region of spacetime. [p. 974]

That is actually the view we wish to endorse. Due to their lack of spacetime localization, almost local observables cannot be real observables. So, one cannot perform any actual measurement of the fictional observable $\bar{C}$. Accordingly, the feigned operation $T_{\bar{C}}$ could not possibly induce any form of act-outcome correlations

\footnotetext{
2 Test functions are infinitely differentiable function on $M$ that, together with their derivatives, tend to zero at infinity faster than the inverse of any power of the Euclidean distance.
}

that has physical significance, even if $\bar{C}$ violates Condition (III) in Arageorgis and Stergiou's theorem. On the basis of the FAPP recipe outlined in the quotations by Halvorson and Clifton in Section 2.2, a measurement of an observable $C$ localized in the region $\mathcal{O}$ can simulate the measurement of any observable contained in the set $\mathcal{R}_{\delta}(\mathcal{O})$, such as $\bar{C}$, in that these measurements are practically indistinguishable. Yet, the only measurement one can physically perform is that of the local observable $C \in \mathcal{R}(\mathcal{O})$, and we know that, in virtue of microcausality, the corresponding operation $T_{C}$ given by the Lüders rule (1) does not change the expectation values of the observables localized in any other region within the causal complement of $\mathcal{O}$. Under the interpretation of almost local observables as mere fictions, relativistic causality is indeed satisfied from a physical point of view.

This also puts us in position to clarify Halvorson and Clifton's approximate localization scheme. Indeed, almost local observables are introduced to circumvent the problem that local observables do not annihilate the vacuum. The role of the fictional observable $\bar{C}$ is to assure that, even though $C \in \mathcal{R}(\mathcal{O})$ cannot annihilate the vector $\Omega$ for any region $\mathcal{O}$, its expectation value computed in the vacuum is arbitrarily small. Specifically, since $\|C-\bar{C}\|<\delta$ and $|\langle\Omega \mid \bar{C} \Omega\rangle|=0$, it follows that $|\langle\Omega \mid C \Omega\rangle-\langle\Omega \mid \bar{C} \Omega\rangle|=|\langle\Omega \mid C \Omega\rangle|<\delta$, where the positive real number $\delta$ can be set as small as one wishes. So, although it is true that, strictly speaking, Condition (I) is not fulfilled by any observable belonging to a local algebra, for all practical purposes one can still maintain the desideratum that a particle detector described by a local observable ought not to detect any particle in the vacuum. But, as we argued, that is not achieved at the cost of envisaging act-outcome correlations over the entire spacetime.

\section{Conclusion}

We discussed and rejected a claim by Arageorgis and Stergiou (2013) that, within an approximate localization scheme developed by Halvorson and Clifton (2002), almost local observables describing particle detectors would entail act-outcome correlations over the entire spacetime, thereby violating relativistic causality. To address the question raised by Arageorgis and Stergiou in the title of their paper, that is "How much local is almost local?", we offer two possible answers: if the meaning of "local" is understood in terms of localization in a bounded region of spacetime, the answer is that almost local observables are not local enough, in that they do not belong to any local algebra; instead, if the meaning of "local" is understood in terms of a locality condition of no superluminal signalling, the answer is that almost local observables are fully local, in that they do not induce any act-outcome correlations. In particular, our analysis suggests that Halvorson and Clifton's approximate localization scheme is a viable proposal to "salvage the appearances" observed in the laboratory, at least within the framework of Algebraic Quantum Field Theory. One can thus restore particle phenomenology without having to give up relativistic causality.

Of course, there still remains the outstanding problem of the ontology of relativistic quantum field theory. One may actually claim, as Halvorson and Clifton do, that the no-go theorems for the impossibility of particle localizability in the strict sense offer a decisive argument against a particle interpretation. Yet, a field interpretation is not immune to criticism either. In particular, Baker (2009) observed that the objection to particle ontology grounded on the above-mentioned theorems can be turned into an argument against field ontology as well. We do not wish to take any stance on this issue here. We only note that Halvorson and Clifton's approximate localization scheme is compatible with a field interpretation. In fact, Buchholz's (2000) construction of the almost local observable $\bar{C}$ annihilating the vacuum by means of 
test functions $f$ can be connected to the underlying Wightman fields generating the correspondence $\mathcal{O} \longmapsto \mathcal{R}(\mathcal{O})$ in Algebraic Quantum Field Theory. In this picture of particle phenomenology with field ontology, the appearance of particles would result from local excitations of a quantum field in Minkowski spacetime.

\section{Acknowledgment}

The author would like to thank Aristidis Arageorgis, Jeremy Butterfield, Adam Caulton, John Earman and Miklos Redei, as well as the philosophy of physics groups in Cambridge and Irvine, for helpful comments and discussions.

\section{References}

Arageorgis, A., \& Stergiou, C. (2013). On particle phenomenology without particle ontology: How much local is almost local? Foundations of Physics, 43(8), 969977.

Baker, D. (2009). Against field interpretations of quantum field theory. British Journal for the Philosophy of Science, 60, 585-609.

Buchholz, D. (2000). Algebraic quantum field theory: A status report. arXiv:org math-ph/0011044.

Busch, P. (1999). Unsharp localization and causality in relativistic quantum theory. Journal of Physics A, 32, 6535-6546.
Busch, P., \& Singh, J. (1998). Lüders theorem for unsharp quantum

measurements. Physics Letters A, 249, 10-12.

Fleming, G., \& Butterfield, J. (1999). Strange positions. In: J. Butterfield, \& C. Pagonis (Eds.), From physics to philosophy (pp. 108-165). New York: Cambridge University Press.

Haag, R. (1992). Local quantum physics (2nd edition). New York: Springer.

Halvorson, H. (2001). Reeh-Schlieder defeats Newton-Wigner: On alternative localization schemes in relativistic quantum field theory. Philosophy of Science $68,111-138$

Halvorson, H., \& Clifton, R. (2002). No place for particles in relativistic quantum theories?. In: M. Kuhlmann, H. Lyre, \& A. Wayne (Eds.), Ontological aspects of quantum field theory (pp. 165-179). Singapore: World Scientific.

Hegerfeldt, G. C. (1998). Instantaneous spreading and Einstein causality in quantum theory. Annals of Physics, 7, 716-725.

Kraus, K. (1983). States, effects and operations, Lecture notes in physics (Vol. 190) New York: Springer.

Malament, D. (1996). Defense of dogma: Why there cannot be a relativistic mechanics of (localizable) particles. In: R. Clifton (Ed.), Perspectives on quantum reality (pp. 1-10). Dordrecht: Kluwer Academic.

Rédei, M., \& Valente, G. (2010). How local are local operations in local quantum field theory? Studies in the History and Philosophy of Modern Physics, 41, 346-353.

Redhead, M. (1995). More Ado about Nothing. Foundations of Physics, 25, 123-137.

Reeh, H., \& Schlieder, S. (1961). Bemerkungen zur unitaraquivalenz von

Lorentzin-varianten Feldern. Nuovo Cimento, 22, 1051-1068.

Shimony, A. (1986). Events and processes in the quantum world. In: R. Penrose, \& C. Isham (Eds.), Quantum concepts in space and time. Oxford: Oxford University Press.

Valente, G. (2014), Does the Reeh-Schlieder theorem violate relativistic causality? forthcoming in History and Philosophy of Modern Physics, http://dx.doi.org/10. 106/j.shpsb.2014.05.006 